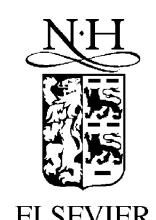

Information Processing Letters 76 (2000) 83-90

\title{
Information
} Processing

\section{Deterministic small-world communication networks}

\author{
Francesc Comellas ${ }^{\mathrm{a}, *}$, Javier Ozón ${ }^{\mathrm{a}}$, Joseph G. Peters ${ }^{\mathrm{b}}$ \\ ${ }^{a}$ Departament de Matemàtica Aplicada i Telemàtica, Universitat Politècnica de Catalunya, Campus Nord C3, J. Girona 1-3, \\ 08034 Barcelona, Catalonia, Spain \\ b School of Computing Science, Simon Fraser University, Burnaby, British Columbia, Canada V5A 1S6 \\ Received 1 January 2000; received in revised form 1 April 2000 \\ Communicated by Dr. S.E. Hambrusch
}

\begin{abstract}
Many real life networks, including the World Wide Web, electric power grids, and social networks, are small-world networks. The two distinguishing characteristics of small-world networks are strong local clustering (nodes have many mutual neighbors), and small average distance between two nodes. Small-world networks are promising candidates for communication networks since typical data-flow patterns in communication networks show a large amount of clustering with a small number of "longdistance" communications that need to be completed quickly.

Most previous research on small-world networks has used simulations, probabilistic techniques, and random replacements of edges to study the limiting behaviour of these networks. In this paper, we initiate the study of small-world networks as communication networks using graph-theoretic methods to obtain exact results. We construct networks with strong local clustering and small diameter (instead of average distance). Our networks have the additional property that they are regular. (C) 2000 Elsevier Science B.V. All rights reserved.
\end{abstract}

Keywords: Small-world networks; Communication networks; Interconnection networks; Distributed systems

\section{Introduction}

Small-world networks were introduced by Watts and Strogatz in a recent paper [9] as models of real world situations including electric power grids, the spread of diseases in populations, the collaboration networks of film actors, and the neural network of the worm Caenorhabditis elegans [8,9]. Other examples include the World Wide Web [1] and the network

\footnotetext{
Research supported by a NATO Collaborative Research Grant (CRG 972241). Additional support for the first two authors provided by CICYT, Spain (TIC97-0963). Additional support for the third author provided by NSERC of Canada.

* Corresponding author.

E-mail address: comellas@mat.upc.es (F. Comellas).
}

of mathematicians with a given Erdôs number [5]. The initial paper has led to considerable research and publication activity [2,6-8].

Many classes of structured ${ }^{1}$ networks, including the restricted class of circulant graphs studied in [9] and subsequent papers, have strong local clustering (nodes have many mutual neighbors), but large average distances between pairs of nodes. The opposite extreme is random networks which have small average distances but exhibit very little clustering. Networks between these two extremes can be constructed by starting with a structured network and randomly

\footnotetext{
${ }^{1}$ In [9] and subsequent papers, the term "regular" is used informally to refer to these networks. We use "structured" instead and use "regular" in its usual graph-theoretic sense.
} 
moving one endpoint of each edge with probability $p$. Structured graphs correspond to $p=0$ and random graphs are obtained by setting $p=1$. Watts and Strogatz discovered that the average distance between nodes (which they called characteristic path length), decreases rapidly as $p$ increases, but clustering decreases slowly. In [9], small-world networks occur in the region around $p=0.01$ (i.e., a random change of only $1 \%$ of the edges). When $p=0.01$, the average distance is approximately $20 \%$ of its original value but $95 \%$ of the clustering remains.

Small-world networks are promising candidates for communication networks since data-flow patterns show a large amount of clustering with a small number of "long-distance" communications that need to be accomplished efficiently. The probabilistic analysis techniques and random replacements of edges that have been used in most previous studies of smallworld networks are not appropriate for communication networks which have fixed interconnections. In this paper, we replace the probabilistic models with deterministic small-world networks and non-random interconnection patterns. Instead of the average distance between nodes, we will study the maximum distance or diameter. Small diameter is consistent with the concept of small-world network and it is easier to calculate. Moreover, it is often more relevant than average distance in the study of communication networks.

We define a parameter $h$ that serves the same purpose that $p$ serves in the probabilistic models and give exact expressions for the diameter and the clustering as functions of this new parameter. Our constructions give networks with small diameters and strong clustering similar to the networks obtained in previous studies with probabilistic methods. In the networks that result from the probabilistic methods, the nodes will have different numbers of neighbors, so the networks are not regular. Our constructions add new edges with the goal of reducing the diameter of the network, but we also replace some edges in a way that restores the regularity. Our model retains the essence of the probabilistic model, but it is more useful for practical applications in which the number of neighbors of a node must be fixed because of technical considerations.

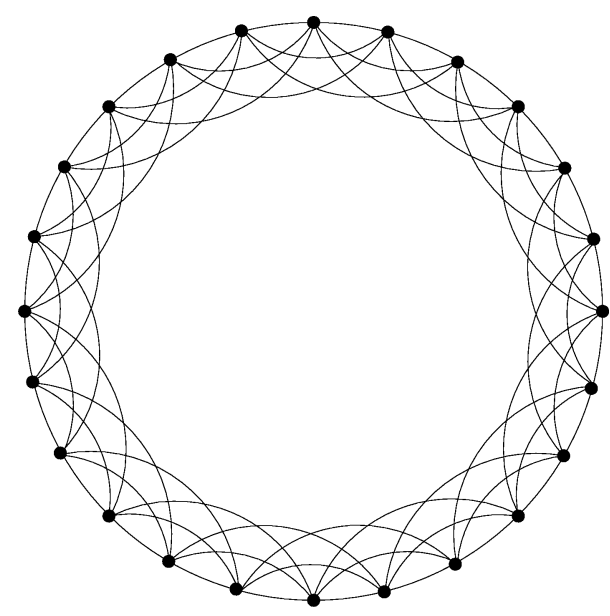

Fig. 1. $C_{n, \Delta}$ with $n=24$ and $\Delta=6$, a circulant graph with steps $\pm 1 ; \pm 2 ; \pm 3$.

\section{Notation}

In this paper a network will be represented by a graph $G=(V, E)$ of order $n=|V|$. We will use standard graph theory terminology. The degree of a node $x$, denoted $\operatorname{deg}(x)$, is the number of edges incident on $x$ and the degree of a graph $G$ is

$\Delta=\max _{x \in V} \operatorname{deg}(x)$.

A graph is $\Delta$-regular if the degree of every node is $\Delta$. The distance between two nodes $x$ and $y, d(x, y)$, is the number of edges of a shortest path between $x$ and $y$. The maximum distance over all pairs of nodes,

$D=\max _{x, y \in V} d(x, y)$,

is the diameter of the graph.

The basic family of graphs considered here (and in [9]) is circulant graphs. The circulant graph $C(n ; 1$, $2, \ldots, \Delta / 2), \Delta$ even, has $n$ nodes labeled with integers modulo $n$, and $\Delta$ links per node such that each node $i$ is adjacent to the nodes $i \pm 1, i \pm 2$, $\ldots, i \pm \Delta / 2(\bmod n)$. We will use $C_{n, \Delta}$ to denote $C(n ; 1,2, \ldots, \Delta / 2)$. This graph has diameter $D=$ $\lceil n / \Delta\rceil$. We will refer to edges between node $i$ and nodes $i \pm \ell, \ell>1$, as chords of length $\ell$. We will also use double loop graphs, $C(n ; a, b)$, which are circulant graphs such that each node $i$ is adjacent to the 
four nodes $i \pm a, i \pm b(\bmod n)$. The diameter of $C(n$; $a, b)$ is

$D=\left\lceil\frac{-1+\sqrt{2 n-1}}{2}\right\rceil$

for $a=D$ and $b=D+1[3,4]$. Other graphs used in this paper are the complete graph on $n$ nodes $K_{n}$, and the star graph $K_{1, n-1}$, which has $n$ nodes and $n-1$ edges joining one node to all the others.

Most previous studies of small-world networks have used two parameters to characterize the networks: average distance and clustering. As explained above, we will study the diameter instead of average distance. We delay the formal definition of clustering until Section 4. Informally, it is the fraction of possible edges among neighbors of a node that are actually present, averaged over all nodes.

\section{Diameter reduction}

The diameter of $C_{n, \Delta}=C(n ; 1,2, \ldots, \Delta / 2), \Delta$ even, is $D_{C_{n, \Delta}}=\lceil n / \Delta\rceil$ and $C_{n, \Delta}$ is $\Delta$-regular. In this section we show how to reduce the diameter of $C_{n, \Delta}$ by selecting a number of nodes of $C_{n, \Delta}$ to be hubs and then using a graph $H$ of known diameter $D_{H}$ to interconnect the hubs. The resulting graph $G$ has a smaller diameter than $C_{n, \Delta}$ but is not $\Delta$-regular because the hubs have acquired more edges. In Section 4, we will show how to modify $G$ in the regions near its hubs to obtain a $\Delta$-regular graph with the same diameter as $G$.

Definition 1. A segment $\mathcal{S}$ of $C_{n, \Delta}$ is the subgraph of $C_{n, \Delta}$ induced by two consecutive hubs $i$ and $j$ and all nodes between $i$ and $j$. The length of $\mathcal{S}$ is $\ell_{\mathcal{S}}=\min (|i-j|, n-|i-j|)$.

The following lemma and theorem establish bounds on the number of hubs that are needed to achieve a given diameter.

Lemma 2. Let $\mathcal{S}$ be a segment of $C_{n, \Delta}, \Delta$ even, with length $(k-1) \Delta+1<\ell_{\mathcal{S}} \leqslant k \Delta+1, k \geqslant 1$. The maximum distance between any node of $\mathcal{S}$ and one of the end nodes of $\mathcal{S}$ is $k$.

Proof. From any given node of $\mathcal{S}$, use chords of length $\Delta / 2$ or $-\Delta / 2$ to make jumps towards the nearest end node of $\mathcal{S}$. The last jump might use a shorter chord. The maximum number of jumps is exactly $k$.

Theorem 3. Given $C_{n, \Delta}, \Delta$ even, and $D<D_{C_{n, \Delta}}$. The number of hubs required to construct a new graph $G$ with diameter at most $D_{G} \leqslant D$ from $C_{n, \Delta}$ by using a graph $\mathrm{H}$ of diameter $D_{H}$ to interconnect the hubs is

$h=\left\lceil\frac{2 n}{\Delta\left(D-D_{H}\right)+2}\right\rceil$ if $D-D_{H}$ is even and

$h=\left\lceil\frac{2 n-2 \Delta}{\Delta\left(D-D_{H}-1\right)+2}\right\rceil$ if $D-D_{H}$ is odd.

Proof. If $D-D_{H}$ is even, we can choose $h$ nodes of $C_{n, \Delta}$ to be hubs such that each segment $\mathcal{S}$ has length $(k-1) \Delta+1<\ell_{\mathcal{S}} \leqslant k \Delta+1$ for some $k \geqslant 1$. Then, by Lemma 2 , the maximum distance from any node of $G$ to a hub is $k$ and the maximum distance between any two hubs of $G$ is $D_{H}$. Thus, the diameter of $G$ is $D_{G} \leqslant 2 k+D_{H}$.

Now, we determine the value of $h$. Let $h=\lceil n /$ $(k \Delta+1)\rceil$. Then all segments are of length at most $k \Delta+1$ and so the diameter is $D_{G} \leqslant 2 k+D_{H}=D$. Since $k=\left(D-D_{H}\right) / 2$, we get $h=\left\lceil 2 n /\left(\Delta\left(D-D_{H}\right)\right.\right.$ $+2)\rceil$.

If $D-D_{H}$ is odd, let $h=\lceil(n-\Delta) /(k \Delta+1)\rceil$. Then $h-1$ of the segments have length $k \Delta+1$ and the length of the remaining segment is $n-(h-1)(k \Delta+1)$ $\leqslant(k+1) \Delta+1$. In this case the diameter is $D_{G} \leqslant$ $(2 k+1)+D_{H}=D$. Since $k=\left(D-D_{H}-1\right) / 2$ we get $h=\left\lceil(2 n-2 \Delta) /\left(\Delta\left(D-D_{H}-1\right)+2\right)\right\rceil$.

Remark 4. The theorem assumes that $D_{H}$ is known. For example, this is the case if $H$ is a complete graph or a star graph. If $D_{H}$ depends on the order of $H$, the theorem can still be used by fixing $D-D_{H}$ as shown in the next example.

Example 5. The diameter of $C_{n, \Delta}$ with $n=1000$ and $\Delta=10$ is 100 . If we want a graph with diameter $D_{G} \leqslant 34$ and we use a complete graph for joining the hubs, then $D_{H}=1$ and $D-D_{H}$ is odd. From Theorem 3, $h=7$ hubs are required. On the other hand, if we use the star graph, then $D_{H}=2$ and $D-D_{H}$ is even. In this case, $h=7$ hubs are also required. Finally, if $H$ is a double loop graph, $D_{H}$ depends on the number of hubs, so we fix $D-D_{H}$. 
To obtain a graph with diameter $D_{G} \leqslant 32+D_{H}$, we need $h=7$ by Theorem 3 . In this case the diameter of the double loop graph is $D_{H}=2$ using chords of length 2 and 3 , so $D_{G} \leqslant 34$.

We now establish bounds on the diameter that can be obtained when the number of hubs is given.

Theorem 6. Given $C_{n, \Delta}, \Delta$ even, let $H$ denote a graph with $h$ nodes and diameter $D_{H}$. There is a graph $G$ with $n$ nodes and $h$ hubs (using graph $H$ to interconnect the hubs) which has diameter $D_{G} \leqslant 2 k+$ $D_{H}$, where $k=\lceil(\lceil n / h\rceil-1) / \Delta\rceil$. If the condition $n-((k-1) \Delta+1)(h-1) \leqslant k \Delta+1$ is also satisfied, then the diameter is $D_{G} \leqslant 2 k-1+D_{H}$.

Proof. Since the number of hubs is $h$, it is possible to divide $C_{n, \Delta}$ into segments of length at most $\lceil n / h\rceil$. Let $k$ be the integer such that $(k-1) \Delta+1<\lceil n / h\rceil \leqslant$ $k \Delta+1$, so $k=\lceil(\lceil n / h\rceil-1) / \Delta\rceil$. Construct graph $G$ by using the graph $H$ to interconnect the hubs of $C_{n, \Delta}$. By Lemma 2, the distance from any node of $G$ to a hub is at most $k$ and the distance between any two hubs of $G$ is at most $D_{H}$, so $D_{G} \leqslant 2 k+D_{H}$.

If $n-((k-1) \Delta+1)(h-1) \leqslant k \Delta+1$, then we can choose the hubs so that $h-1$ of the segments have length $(k-1) \Delta+1$ and the remaining segment has length at most $k \Delta+1$. By Lemma 2 , the distance to a hub is at most $k-1$ for any node in $h-1$ of the segments and at most $k$ for nodes in the remaining segment. This gives $D_{G} \leqslant 2 k-1+D_{H}$.

Remark 7. It is not possible to give equalities in the expressions for the diameter in Theorem 6 because the final diameter depends on the structure of the graph $H$ that is used to join the hubs. For example, suppose that $h-1$ segments have length $(k-1) \Delta+1$ and the remaining segment has length at most $k \Delta+1$. If the two hubs of the longer segment are at distance less than $D_{H}$ from all of the other hubs, then $D_{G} \leqslant$ $2 k-2+D_{H}$.

Example 8. The diameter of $C_{n, \Delta}$ with $n=1000$ and $\Delta=4$ is 250 . Using $h=99$ hubs, the diameter can be reduced to $D_{G} \leqslant 2 k+D_{H}$ with $k=3$ by Theorem 6 .
On the other hand, Theorem 3 shows that the same diameter $D_{G} \leqslant 2 k+D_{H}$ is possible with

$h=\left\lceil\frac{2 n}{\Delta\left(D-D_{H}\right)+2}\right\rceil=77$

hubs. In this case, 76 of the segments have length $k \Delta+1=13$ and the remaining segment has length 12 .

Example 9. The diameter of $C_{n, \Delta}$ with $n=1000$ and $\Delta=10$ is 100 . If we want a graph with diameter $D_{G} \leqslant$ $64+D_{H}$ then $h=4$ hubs are required by Theorem 3 . However, with 4 hubs the diameter can be reduced to $D_{G} \leqslant 50+D_{H}$ using Theorem 6 and

$k=\left\lceil\frac{\lceil n / h\rceil-1}{\Delta}\right\rceil=25$.

There are many choices for the graph $H$ that joins the hubs. For a given number of hubs $h$, the complete graph $K_{h}$ gives the maximum reduction in the diameter, but also causes the maximum increase in the degree of the hubs. If the maximum degree is not important, then a good choice is the star graph $K_{1, h-1}$, which has diameter 2 and uses the minimum possible number of edges to connect one hub to all the others. An interesting choice for $H$ is the family of double loop graphs $C(h ; a, b)$. The diameter $D=\lceil(-1+\sqrt{2 h-1}) / 2\rceil$ is relatively small and the degree is only 4 (see [3]). Consider $C_{10000,10}$, which has diameter 1000 , and $h=50$ hubs. The diameter is reduced to 45 using a double loop graph compared to a diameter of 41 using $K_{50}$, but the degree of the hubs is only increased by 4 instead of by 49 with $K_{50}$. Other families of regular low degree graphs with known diameters can be used for $H$. We will restrict our attention to double loop graphs in this paper because they preserve important properties of the transformed graphs and permit easy derivations of the parameters of small-world networks. Note that in this particular example, the diameter can be reduced from 1000 to 41 (a factor close to 25) while only affecting 50 of the 10000 nodes (only $0.5 \%$ of the nodes) of the initial graph.

When $D_{G} \gg D_{H}$, we obtain

$h \simeq\left\lceil\frac{2 n}{\Delta\left(D_{G}-D_{H}\right)+2}\right\rceil \simeq\left\lceil\frac{2 n}{\Delta D_{G}}\right\rceil \simeq 2 \frac{D_{C_{n, \Delta}}}{D_{G}}$

from Theorem 3. Thus, if we want to reduce the diameter by a factor of 10 we will need approximately 
20 hubs and this value does not depend on the order of the initial graph. So, even if the number of nodes is $10^{7}$, a reduction of the diameter from 100 to 10 will only require approximately 20 hubs. These results show that it is possible to dramatically reduce the diameter of a network while affecting only a very small fraction of its nodes.

\section{Clustering and regularity}

Clustering is a measure of the connectedness of a graph and is one of the parameters used to characterize small-world networks. In this section, we calculate the clustering parameters of $C_{n, \Delta}$ and the graphs that result when hubs of $C_{n, \Delta}$ have been identified and interconnected and some small modifications have been made to preserve regularity.

Definition 10. For each node $i$ of a graph $G$, let $n_{i}$ be the number of neighbors of $i$. Let $\mathcal{C}_{i}$ be the fraction of the $n_{i}\left(n_{i}-1\right) / 2$ possible edges among the neighbors of $i$ that are present in $G$. The clustering parameter of $G$, denoted $\mathcal{C}_{G}$, is the average over all nodes $i$ of $\mathcal{C}_{i}$.

Note that the clustering parameter can be calculated from the number of cycles of length three (triangles) in which each given node is included.

We start by calculating the clustering parameter for the circulant graph $C_{n, \Delta}$ before any hubs are added and interconnected.

Proposition 11. The clustering parameter of $C_{n, \Delta}$ is

$\mathcal{C}_{C_{n, \Delta}}=\frac{3(\Delta-2)}{4(\Delta-1)}$.

Proof. Since $C_{n, \Delta}$ is node-symmetric, each node contributes equally to the clustering parameter and the calculations are the same for each node $i$. First we calculate the number of triangles to which node $i$ belongs. Nodes $i$ and $i+1$ have $\Delta-2$ neighbors in common; nodes $i$ and $i+2$ have $\Delta-3$ common neighbors, and so on. In general, nodes $i$ and $i+j$ have $\Delta-(j+1)$ common neighbors, $1 \leqslant j \leqslant \Delta / 2$, and the same is true for nodes $i$ and $i-j$. Summing over all neighbors of node $i$ gives

$$
\frac{1}{2} \cdot 2 \cdot \sum_{j=1}^{\Delta / 2}(\Delta-(j+1))=\frac{3}{4} \Delta\left(\frac{\Delta}{2}-1\right)
$$

triangles containing node $i$. The factor $\frac{1}{2}$ appears because each triangle is counted twice.

The maximum possible number of edges among the neighbors of a node $i$ is $\Delta(\Delta-1) / 2$ and the number of these edges that are present in $C_{n, \Delta}$ is the same as the number of triangles to which $i$ belongs. Therefore, the clustering parameter of $C_{n, \Delta}$ is

$\mathcal{C}_{C_{n, \Delta}}=\frac{3}{4} \Delta\left(\frac{\Delta}{2}-1\right) / \frac{\Delta(\Delta-1)}{2}=\frac{3(\Delta-2)}{4(\Delta-1)}$.

In Section 3, we showed how to reduce the diameter of $C_{n, \Delta}$ by selecting $h$ nodes to be hubs and then interconnecting the hubs with a graph $H$ with $h$ nodes and diameter $D_{H}$. The resulting graph $G$ is not $\Delta$-regular because the hubs have acquired more edges. In particular, if $H$ is a double loop graph $C(h ; a, b)$, then the degree of the hubs is $\Delta+4$. We will now show how to modify $G$ to obtain a $\Delta$-regular graph $G^{\prime}$ with the same diameter as $G$. Then we will determine the clustering parameter of $G^{\prime}$.

To obtain a $\Delta$-regular graph $G^{\prime}$ from $G$, four edges incident on each hub must be removed. This will reduce the degrees of the other endpoints of the removed edges to $\Delta-1$, so two edges need to be added to obtain $\Delta$-regularity (see Fig. 2 ). We must do this in a way that avoids multiple edges, so the added edges should not be between nodes which are adjacent in $G$. We also want to maintain the same diameter and minimize the change in the clustering parameter.

Suppose that node $i$ is a hub. The edges from $i$ to $i \pm \Delta / 2$ should not be removed if there is some segment with length $\ell_{\mathcal{S}}=k \Delta+1$ because this will increase the diameter (see Theorem 6). On the other hand, we prove in the next Lemma that any removal of four edges around a given hub (affecting two nodes on each side as in Fig. 2) leads to the same reduction in the number of triangles. We choose two nodes on each side to ensure that the nodes that will be connected by new edges are not adjacent in $C_{n, \Delta}$.

Lemma 12. Let $i$ be a node of $C_{n, \Delta}$. Removing the edges $(i, i \pm(\Delta / 2-1))$ and $(i, i \pm(\Delta / 2-2))$ or any other combination that affects two nodes on each side of $i$, and connecting the nodes as in Fig. 2 reduces the number of triangles by $2 \Delta-6$. We assume that the affected nodes are not adjacent in $C_{n, \Delta}$. 


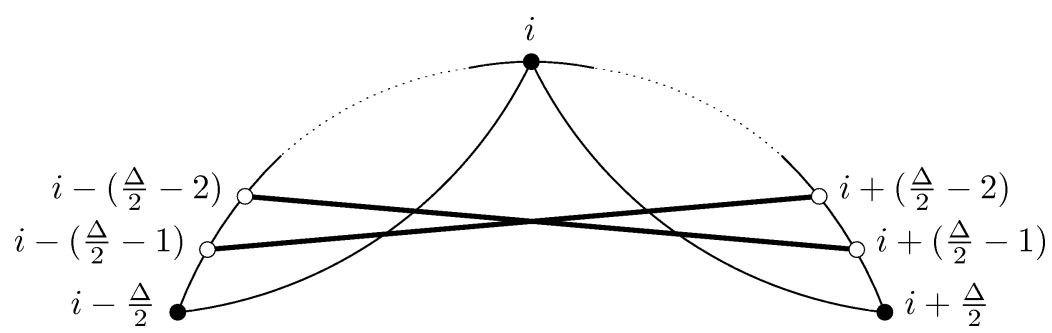

Fig. 2. The reconnection.

Proof. Choose nodes $i-j, i-k, i+l, i+m$ such that $1 \leqslant j, k, l, m \leqslant \Delta / 2, j \neq k, l \neq m$. The number of removed triangles on one side is $\Delta-(j+1)+\Delta-$ $(k+1)-1$ (see the proof of Proposition 11). Note that we subtract 1 because one triangle has been counted twice. The corresponding value for the other side is $\Delta-(l+1)+\Delta-(m+1)-1$. Thus the total number of removed triangles is $4 \Delta-(j+k+l+m)-6$. On the other hand, when adding the new edges we add some new triangles. If we add an edge between $i-j$ and $i+l$, the number of new triangles is $\Delta / 2-$ $j+\Delta / 2-l$ (i.e., the number of common neighbors of $i-j$ and $i+l$ ). If we connect $i-k$ with $i+m$, the number is $\Delta / 2-k+\Delta / 2-m$ giving a total of $2 \Delta-(j+k+l+m)$ added triangles. This value is the same if we add edges between $i-j$ and $i+m$, and between $i-k$ and $i+l$. Therefore the number of triangles is reduced by $4 \Delta-(j+k+l+m)-$ $6-(2 \Delta-(j+k+l+m))=2 \Delta-6$ and this does not depend on the choice of $j, k, l, m$ as long as no duplicate edges are added.

Consistent with the requirement that edges must be added between nodes which are not adjacent in $C_{n, \Delta}$, we consider the removal of the edges between $i$ and the four nodes $i \pm(\Delta / 2-1)$ and $i \pm(\Delta / 2-2)$. If we join these 4 nodes with edges as shown in Fig. 2, then the condition $(\Delta / 2-1)+(\Delta / 2-2)>\Delta / 2$ is necessary to avoid duplicate edges. In this case $\Delta>6$. Any other selection of nodes or another way of connecting the four nodes will require $\Delta>8$ if $i \pm \Delta / 2$ cannot be chosen. On the other hand, when all segments have length at most $\ell<k \Delta+1$ then it is possible to remove the edges from $i$ to $i \pm \Delta / 2$ without affecting the diameter. In this case the result of Lemma 12 is true for $\Delta>4$.
Note that in the case that all segments have length at most $\ell \leqslant \Delta+1$ (which corresponds to $k=1$ in Theorem 6) the bound on the diameter is increased (by 2). This is because the distance of any node of $G$ to its nearest hub is $k=1$ and we have removed the edge in $G^{\prime}$ that connects the affected nodes to the hub (so we have nodes in $G^{\prime}$ at distance 2 from the nearest hubs). In any other case (i.e., $k \geqslant 2$ ), the final diameter does not change.

To calculate the clustering parameter, we also need to take into account the triangles induced by the graph $H$. If $H$ is a double loop graph and the number of hubs is greater than 8 , there is no contribution from $H$.

Let $C_{n, \Delta}^{\prime}$ denote the graph resulting from the graph $C_{n, \Delta}$ after choosing $h$ hubs, connecting them with a double loop graph, and modifying the connections according to the above rules to regain regularity.

Proposition 13. The clustering parameter of $C_{n, \Delta}^{\prime}$ with $h \geqslant 8$ hubs is

$\mathcal{C}_{C_{n, \Delta}^{\prime}}=\mathcal{C}_{C_{n, \Delta}}-\frac{h}{n} \frac{6(2 \Delta-6)}{\Delta(\Delta-1)}$,

where $\mathcal{C}_{C_{n, \Delta}}=3(\Delta-2) / 4(\Delta-1)$ is the clustering parameter of $C_{n, \Delta}$.

Proof. Let $\mathcal{T}_{i}$ denote the number of triangles of a node $i$ and let $\mathcal{T}_{\mathcal{G}}$ denote the number of triangles of a graph $\mathcal{G}$.

$$
\begin{aligned}
\mathcal{C}_{C_{n, \Delta}^{\prime}} & =\frac{1}{n} \sum_{i} \mathcal{C}_{i}=\frac{1}{n} \sum_{i} \frac{\mathcal{T}_{i}}{\Delta(\Delta-1) / 2} \\
& =\frac{1}{n}\left(\frac{1}{\Delta(\Delta-1) / 2}\right) 3 \mathcal{I}_{C_{n, \Delta}^{\prime}} .
\end{aligned}
$$


The total number of triangles is the total from $C_{n, \Delta}$ minus $h(2 \Delta-6)$ according to Lemma 12 , so the clustering parameter of $C_{n, \Delta}^{\prime}$ is

$$
\begin{aligned}
\mathcal{C}_{C_{n, \Delta}^{\prime}} & =\frac{3 \mathcal{T}_{C_{n, \Delta}}-3 h(2 \Delta-6)}{n \Delta(\Delta-1) / 2} \\
& =\mathcal{C}_{C_{n, \Delta}}-\frac{h}{n} \frac{6(2 \Delta-6)}{\Delta(\Delta-1)} .
\end{aligned}
$$

Proposition 13 can be used together with Theorem 6 to show that $C_{n, \Delta}^{\prime}$ is a small-world network. Consider $C_{1000,10}$ with $h=50$ hubs. The diameter of $C_{1000,10}$ is $D_{C_{1000,10}}=100$ and the clustering parameter is $\mathcal{C}_{C_{1000,10}}=0.667$. The diameter of $C_{1000,10}^{\prime}$ is $D_{C_{1000,10}^{\prime}}=9$ by Theorem 6 and the clustering parameter is $\mathcal{C}_{C_{1000,10}^{\prime}}=0.62$ by Proposition 13. So, the diameter of $C_{1000,10}^{\prime}$ is only $9 \%$ of the diameter of $C_{1000,10}$ while the clustering parameter of $C_{1000,10}^{\prime}$ remains nearly the same at $93 \%$ of the value for $C_{1000,10}$.

\section{Comparison of analytical and numerical approaches}

In Fig. 3, we compare our analytical results with numerical values obtained using the method of Watts and Strogatz [9] and an initial circulant graph $C_{1000,10}$. The numerical clustering and diameter curves in the figure were obtained by using the technique of [9] and averaging the results of 20 runs. The double loop clustering and diameter curves are for the graphs $C_{1000,10}^{\prime}$ obtained using the methods of this paper and double loop graphs to interconnect the hubs. The parameter $p$ for the numerical results corresponds to the ratio of the number of added and modified edges to the total number of edges in our analytical models. For $C_{n, \Delta}^{\prime}$, the ratio is $4 h /|E|$. In this example, $|E|=n \Delta / 2=5000$ and $p=4 h / 5000$. The fifth curve in Fig. 3 shows the diameter when star graphs $K_{1, h-1}$ are used to interconnect $h$ hubs of $C_{1000,10}$. In this case, no modifications were made to recover the regularity of the resulting graphs, so $p=(h-1) /|E|$. All of the curves are normalized with respect to the graph $C_{1000,10}$; they show the diameters and clustering parameters as fractions of the values at $p=0$.

The small-world region in Fig. 3 occurs around $p=$ 0.01 where clustering remains above $95 \%$ of the value for $p=0$ and diameter is less than $20 \%$ of the value for $p=0$. In our graph-theoretic models, $p=0.01$ corresponds to $h=12$ hubs when double loop graphs are used to interconnect the hubs and $h=51$ when star graphs are used. The figure shows that smallworld behaviour is more pronounced in our graphtheoretic models than in the probabilistic model. Since the hubs are chosen exactly rather than at random, the

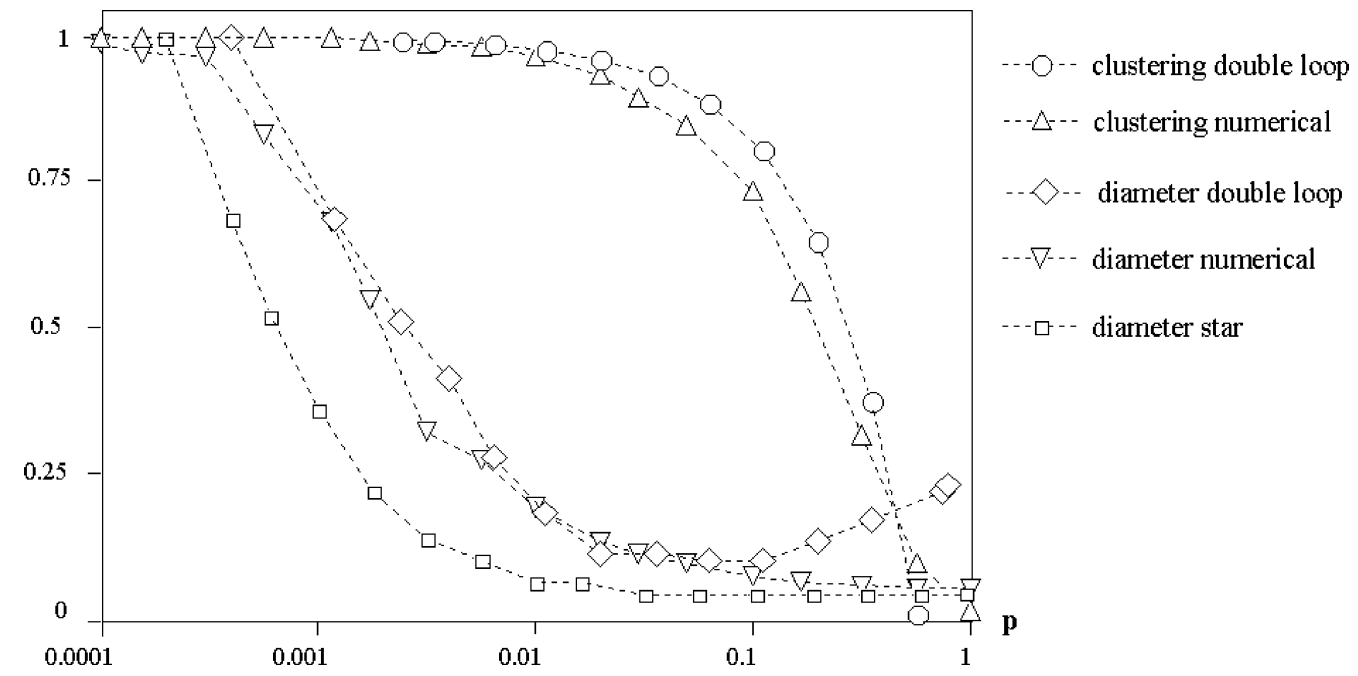

Fig. 3. Comparison of numerical and analytical clustering and diameter. 
clustering around the small-world region is greater in our model while the diameter is smaller.

We also see that the diameter decreases more quickly in our model with star graphs than with double loop graphs. This is because the star graph uses the smallest possible number of edges to join the hubs and always has diameter 2 , whereas the diameter of the double loop graph increases with its order. Finally, for the double loop graphs in our example, the diameter of $C_{n, \Delta}^{\prime}$ begins to increase around $p=0.08$ where the segments have length at most $\Delta+1$ and the distance from any node to a hub is 1 . Adding more hubs increases the diameter of the double loop graph but the distance from any node to the nearest hub remains 1 . Nevertheless, this increase of the diameter occurs far from the small-world region.

\section{References}

[1] R. Albert, H. Jeong, A.-L. Barabási, Diameter of the world wide web, Nature 401 (1999) 130-131.
[2] M. Barthelemy, L.A.N. Amaral, Small-world networks: Evidence for a crossover picture, Phys. Rev. Lett. 82 (15) (1999) 3180-3183.

[3] J.-C. Bermond, F. Comellas, D.F. Hsu, Distributed loop computer networks: A survey, J. Parallel Distributed Comput. 24 (1995) 2-10.

[4] F.T. Boesch, J.K. Wang, Reliable circulant networks with minimum transmission delay, IEEE Trans. Circuits Systems CAS-32 (1985) 1286-1291.

[5] R. de Castro, J.W. Grossman, Famous trails to Paul Erdős, Math. Intelligencer 21 (3) (1999) 51-63.

[6] H. Herzel, How to quantify 'small-world' networks?, Fractals 6 (4) (1998) 301-303.

[7] S.A. Pandit, R.E. Amritkar, Characterization and control of small-world networks, Phys. Rev. E 60 (2) (1999) R1119R1122.

[8] D.J. Watts, Small Worlds: The Dynamics of Networks between Order and Randomness, Princeton University Press, Princeton, NJ, 1999.

[9] D.J. Watts, H. Strogatz, Collective dynamics of 'small-world' networks, Nature 393 (1998) 440-442. 